\title{
Nuevas adiciones de angiospermas a la flora del Perú
}

\author{
Angiosperms additions to flora of Peru
}

\author{
Eric F. Rodríguez ${ }^{1}$, Rodolfo Vásquez ${ }^{2}$, Rocío Rojas², Gloria Calatayud ${ }^{2}$, Blanca \\ León $^{3}$ y José Campos ${ }^{4}$
}

\begin{abstract}
${ }^{1}$ Herbarium Truxillense (HUT), Universidad Nacional de Trujillo, Jr. San Martín 392, Trujillo- Perú. E-mail:

efrr@unitru.edu.pe

2Jardín Botánico de Missouri - Oxapampa, Pasco \& Cusco, Perú. E-mail: jbmperu@speedy.com.pe ${ }^{3}$ Plant Resources Center, University of Texas at Austin, 1 University Station F0404, Austin, TX 787120471, U.S.A. \& Museo de Historia Natural de la U.N.M.S.M.. Av. Arenales 1256, Aptdo. 14-0434, Lima-14, Perú. E-mail:

blanca.leon@mail.utexas.edu ${ }^{4}$ Ex-Investigador del Proyecto Flora del Perú. E-mail: joricampos@yahoo.es
\end{abstract}

Presentado: 23/11/2005 Aceptado: $\quad 07 / 06 / 2006$

\section{Resumen}

Se da a conocer 131 nuevas adiciones de Angiospermas a la Flora Peruana procedentes principalmente del norte del Perú, Departamentos de Amazonas (Prov. Bagua y Condorcanqui) y Cajamarca (Prov. San Ignacio). Esta nueva contribución al conocimiento de la flora del país es el resultado del trabajo de campo y de herbario de los autores desde 1993 hasta el 2002, en un esfuerzo conjunto entre el Herbarium Truxillense (HUT) y el Herbario del Missouri Botanical Garden (MO) en el marco del Proyecto Flora del Perú. La contrastación específica se realizó con las especies documentadas en el "Catálogo de las Angiospermas y Gimnospermas del Perú" (Brako \& Zarucchi, 1993) y "Diez años de adiciones a la flora del Perú: 1993-2003" (Ulloa Ulloa et al., 2004). Los taxones encontrados se presentan en tres categorías: 18 especies nuevas, seis cambios taxonómicos, 107 nuevos registros, es decir especies que amplían su distribución geográfica hacia el Perú. Considerando las especies nuevas y los nuevos registros, esta contribución adiciona 125 especies a la Flora Peruana.

Palabras Clave: Flora del Perú, especies nuevas, nuevos registros.

\section{Abstract}

We present here 131 new additions to the angiosperm flora of Peru from recent collections in the north of Peru, mostly from department of Amazonas (provinces of Bagua and Condorcanqui) and department of Cajamarca (province of San Ignacio). This new contribution is the result of field and herbarium studies by the various authors in this region from 1993 to 2002, and represents the combined effort of personnel from the Herbarium Truxillense (HUT) and the Herbarium of the Missouri Botanical Garden (MO) as part of the Flora of Peru Project. The species reported here were compared against the list of species documented in the "Catalogue of Flowering Plants and Gymnosperms of Peru» (Brako \& Zarucchi, 1993) and «Ten years of additions to the flora of Peru: 19932003» (Ulloa Ulloa et al., 2004). The new taxa are organized in three categories: 18 species new to science, six new nomenclatural combinations and 107 new records, thereby enlarging their geographical distribution towards to Peru. Considering the new species and the new records, this contribution adds 125 species to the Peruvian Flora.

Keywords: Flora of Peru, News species, news records.

\section{Introducción}

Actualmente existen dos documentos importantes que dan a conocer las especies de la Flora del Perú: E 1 C atálogo de las A ngiospermasy G imnospermas del Perú por Brako \& Zarucchi (1993) con 17,143 especies y D iez A ños de A diciones a la Flora del Perú: 1993-2003 por Ulloa Ulloa et al. (2004) con un registro neto de 1,509 especies, haciendo un total de 18,652 especies de plantas con semillas. Sin embargo, las adiciones a la Flora de este país siguen incrementándose aceleradamente gracias ala exploración del territorio y estudios sistemáticos. Los autores del presente trabajo, han venido contribuyendo al conocimiento de la flora peruana mediante diversas investigaciones, la mayor parte de ellas como resultado del Programa Flora del Perú desarrollado por el Missouri Botanical Garden (MO) (Vásquez, 1997; Vásquez et al., 2002b). Así mismo, los resultados preliminares de estas investigaciones se han venido dando a conocer en diversas reuniones científicas (Vásquez et al., 1998; Vásquez et al., 1999; Vásquez et al., 2002a; Rodríguez et al., 2004). Uno delos trabajos recientes, $\mathrm{N}$ uevas A diciones de A ngiospermas a la Flora Peruana prooedentedela Provincia deSan Ignacio, D pto. C ajamarca (Rodríguez etal., 2004), fue expuesto en el X Congreso Nacional de Botánica (X CONABOT), Trujillo-Perú en mayo del 2004, allí se dieron a conocer 175 nuevas adiciones como resultado del trabajo decampo y herbario para esta Provincia. Parte de las especies han sido dadas a conocer por Ulloa Ulloa et al. (2004), las restantes son presentadas en este documento. De igual manera, como consecuencia del estudio del material botánico procedente de las Provincias de Bagua y Condorcanqui (D pto. Amazonas, Perú) con motivo del tratamiento de la obra Flora dela Cuenca del C enepa y Á reas A dyaœntes (en prep.) y de otras investigaciones pertenecientes a los autores, se han encontrado nuevas adiciones no incluidas para la flora del país. Entonces, agregando los resultados referidos sólo a las especies nuevas y registros nuevos a los existentes en la Flora del Perú, totalizan 18,777 especies. Por ello, a fin de seguir contribuyendo al mejor conocimiento de la Flora Peruana, el objetivo del presente trabajo es dar a conocer estas nuevas adiciones pertenecientes a las Angiospermas procedentes principalmente del Norte del Perú. 


\section{Material y Métodos}

El material estudiado corresponde a las colecciones efectuadas por los autores en las diversas expediciones botánicas realizadas a los D epartamentos de Cajamarca (Prov. de San Ignacio) y Amazonas (Prov. Bagua y Condorcanqui), en el marco del Programa Flora del Perú desarrollado por el Missouri Botanical Garden (MO) y el Herbarium Truxillense (HUT) entre los años 1993 y 2002, excepcionalmente se extiende a otras colecciones y departamentos.

Todas las colecciones citadas están registradas y depositadas en porlo menos uno de los herbarios peruanos: AMAZ, CUZ, HUT, MOL y/ o USM, con duplicados en MO (acrónimos en Holmgren etal., 1990), salvo indicación contraria. Paralelo a las colecciones de herbario se fijó y conservó material en líquido (alcohol etílico 70\% o AFA) para estudiar la estructura floral y tricomas de algunas especies o para dilucidarlas con otros taxa.

Las determinaciones taxonómicas fueron efectuadas estrictamente en el herbario HUT por los autores y por diferentes especialistas que han visitado esta institución. Además, se revisó material incluidos los tipos, en los herbarios siguientes: F, HAO, HUT, NY, MO, MO L, QCNE, USy USM. Adicionalmente, se contrastó la información con la Base de Datos Tropicos del Missouri Botanical Garden Herbarium [MO, http:/ / mobot.mobot.org/ W 3T/ Search/ vast.html]. A simismo, los datos presentados se encuentran en la Base de $D$ atos computarizados del Herbarium Truxillense (HUT) de la Universidad Nacional de Trujillo. Las especies del norte del Perú incluidas aquí, se encuentran restringidas a la zona fitogeográfica de AmotapeHuancabamba (Weigend, 2002, 2004).

Los taxones están distribuidos en tres categonías, a partir de las publicaciones de Brako \& Zarucchi (1993) y Ulloa Ulloa et al. (2004): Especies nuevas descritas con material del área $(*)$, combinaciones taxonómicas nuevas para nombres de las especies listadas en el Catálogo de la Flora del Perú (ł), y las especies restantes son registros nuevos para el País, es decir que están presentes en otras Floras y por ende amplían su distribución geográfica.

Seincluye, la literatura original sólo para el caso de las especies nuevas (para el resto de especies ver International Plant Names Index [IPNI, http:/ / www.ipni.org]); signos y abreviaturas para: Basónimos (B), Sinónimos (S), Nombres principales listados en el Catálogo de la Flora del Perú (†). Seguido a las citaciones de ejemplares (E) se encuentrala distribución geográfica, citándose en forma abreviada los nombres de los departamentos del Perú [AM=Amazonas, $\mathrm{CA}=$ Cajamarca, $\mathrm{CU}=\mathrm{Cuzco}, \mathrm{HU}=\mathrm{Huánuco}$, JU=Junín, LA=Lambayeque, LL=La Libertad, LO =Loreto, $\mathrm{MD}=$ Madre de D ios, $\mathrm{PA}=$ Pasco, $\mathrm{PI}=$ Piura, $\mathrm{SM}=$ San Martín], a continuación el nombre del país(-es) cuando se requiera, luego si la distribución se limita al Perú se anota como Endémicay si es muy amplia como Cosmopolita. Las familias reconocidas se encuentran en orden alfabético y son las aceptadas por Judd et al. $(1999,2002)$ y APG (2003).

\section{Resultados}

Se presentan 131 nuevas adiciones a la Flora del Perú; las cuales están distribuidas en 18 especies nuevas, 6 cambios taxonómicos y 107 registros nuevos de especies que amplían su distribución geográfica hacia el Perú. Considerando las especies nuevas y los nuevos registros, esta contribución adiciona 125 especies a la Flora Peruana.

\section{ACANTHACEAE}

\section{Dicliptera tweediana Nees}

S: D idiptera deltica Bridar., D icliptera lutea Bridar., D iapedium tweedianum (Nees) Kuntze

E: CA, Prov. San Ignacio: R. V ásquez et al. 20143, 20495. Argentina, Bolivia, Brasil (Tipo), Paraguay, Uruguay.

\section{Hygrophila costata Nees}

S: H ygrophila atricheta Bridar., H ygrophila conferta Nees, H ygrophila guianensis Nees, H ygrophila rivularis (Schltdl.) Nees, Ruellia brasiliensis Spreng., Ruellia rivularis Schltdl., Ruellia verticillata Spreng.

E: CA, Prov. San Ignacio: J. Campos \& P. D íaz 2668. Bolivia, Brasil (Tipo), Colombia, Ecuador, Paraguay. Meso América.

\section{Pseuderanthemum hookerianum (Nees) V. M. Baum}

B: Thyrsacanthus hook erianus Nees

S: 0 dontonema hook erianum (Nees) Kuntze

E: CA, Prov. San Ignacio: E. Rodríguez 1699; J. Campos et al. 4223. Ecuador.

\section{ALST ROEMERIACEAE}

4. $\quad$ *Bomarea alstroemeroides Hofreiter \& E. Rodr., Arnaldoa 11(2): 21-27, f. 1a-c, 2c. 2004 [2005].

E: AM, Prov. Chachapoyas: E . Rodríguez et al. 2167a (Holótipo: HUT). Endémica.

\section{AN NONACEAE}

\section{5. ${ }^{*}$ Cremastosperma bullatum Pirie, Arnaldoa}

11(2): 8-10, f.1, 3-5. 2004

E: AM, Prov. Bagua: M. Pirie etal. 71 (Holótipo: U, Isótipos: AAU, AMAZ, CUZ, E, F, HAO, HUT, US, USM, WU), E. Rodríguez et al. 1152 (U), R. V ásquez et al. 24891 (U).

\section{6. ${ }^{*}$ Cremastosperma cenepense Pirie \& Zapata,} Arnaldoa 11(2):13-14, f.2, 9. 2004

E: AM, Prov. Condorcanqui: R. Rojas et al. 269 (Holótipo: U, Isótipos: AMAZ, HUT, MO, USM), R. R ojas et al. 255 (HUT, MO, U, USM)

\section{7. *Cremastosperma yamayakatense Pirie, Arnaldoa 11(2): 10-13 f.2, 6-8. 2004}

E: AM, Prov. Bagua: M. Pirie etal. 57 (Holótipo: U, Isótipos: CUZ, HAO, HUT, K, MO, NY, USM), E . Rodríguez 724 (HUT), Prov. Condorcanqui: R. V ásquez et al. 19055 (MO)

\section{AQUIFOLIACEAE}

8. Ilex obtusata Triana \& Planch. 
E: CA, Prov. San Ignacio: E. Rodríguez \& J. C ampos 1823; J. Campos et al. 4283; J. Campos et al. 4301; E . Rodríguez et al. 2829 (HUT, LOJA, MO, Q CNE, USM).

\section{ARACEAE}

9. Rhodospatha densinervia Engl. \& K. Krause

S: Rhodospatha macrophylla Sodiro

E:CA, Prov. San Ignacio:J. Camposetal. 4167. Ecuador(Tipo), Colombia.

\section{Stenospermation zeacarpium Madison}

E: CA, Prov. San Ignacio: R. V ásquez et al. 20486, 26729; J. Campos \& S. Corrales $3760 ; \mathrm{J}$. Campos \& E. Rodríguez 2822; E. Rodríguez \& M. N uñez 1916. AM, AY, CU, HU, JU, LO, PA, SM. Bolivia, Ecuador (Tipo).

Nota: Nombre nomenclaturalmente correcto de la reciente especie descrita como Stenospermation killipii Croat \& L. D. Gómez, Novon 15(1): 97-99, f. 6d, 7a, b. 2005.

\section{ARECACEAE}

\section{Aiphanes eggersii Burret}

E:TU, Prov. Tumbes: C. D íaz et al. 5837 (HUT, MO), 7521 (MO). Ecuador (Tipo).

\section{ASCLEPIADACEAE}

\section{Oxypetalum erianthum Decne.}

S: G othofreda eriantha (D ecne.) Kuntze

E: CA, Prov. San Ignacio:J. Campos etal. 3874;J. C ampos \& Z . G arća 3968; S. L eiva et al. 1598 (HAO, MO). AM, JU, HU, SM. Bolivia, Brasil (Tipo), Ecuador, Paraguay.

\section{AST ERACEAE}

13. Barnadesia parviflora Spruce ex Benth. \& Hook. f.

S: Barnadesia trianae Hieron.

E: CA, Prov. San Ignacio: J. C ampos \& Z Z G arća 4498. Ecuador, Colombia.

\section{Bidens odorata Cav.}

S: Bidens pilosa fo. odorata (Cav.) Sherff., †Bidens abadiaeDC

E: CA, Prov. San Ignacio: J. C ampos et al. 2769. Bolivia, Colombia, Ecuador, G uatemala, México.

\section{Clibadium microcephalum S. F. Blake}

E: CA, Prov. San Ignacio: E . Rodríguez \& P. Reyes 1729. AM. Ecuador (Tipo), Costa Rica.

\section{Koanophyllon stipuliferum (Rusby) R. M. King} \& H. Rob.

B: E upatorium stipuliferum Rusby

E: CA, Prov. San Ignacio: J. Campos \& 0. D íaz 2379. CU. Bolivia (Tipo del basónimo).

\section{Lepidaploa ehretiifolia (Benth) H. Rob.}

B: $V$ ernonia ehretiifolia Benth

S: Cacalia ehretiaefolia (Benth) Kuntze, V ernonia acuta N.E.Br.

E: CA, Prov. San Ignacio: J. C ampos \& P. D íaz 4406. Venezuela.

18. Lepidaploa trilectorum (Gleason) H. Rob.

B: $V$ ernonia trilectorum Gleason

E: CA, Prov. San Ignacio: J. Campos \& S. N uñez 4183. AM, Prov. Luya: J. Campos \& L . Campos 3104.

\section{Mikania lindbergii Baker}

S: W illoughbya lindbergii (Baker) Kuntze

E: CA, Prov. San Ignacio: E. Rodríguez \& J. Campos 1819. Bolivia, Brasil (Tipo).

\section{Parthenium hysterophorus $\mathrm{L}$.}

S: Parthenium lobatum Buckley, Parthenium pinnatifidum Stokes, A rgyrochaeta parvillora Cav.

E: CA, Prov. San Ignacio: J. Campos \& O D D íaz 2261, 2394. América Boreal y Austral.

\section{Verbesina ampla $\mathrm{M}$. E. Jones}

E: CA, Prov. San Ignacio:J. Campos \& Z . G aráa 3976. México (Tipo).

\section{Vernonanthura diffusa (Less.) H. Rob.}

B: $V$ ernonia diffusa Less.

S: $\mathrm{V}$ ernonia cinerea Less.

E: CA, Prov. San Ignacio: J. Campos \& Z. G aráa 4524; J. Campos\& O.Cano 4694.

\section{Wedelia tambilloana B. L. Turner}

S: A spilia lanceolata S.F. Blake, A spilia jelskii S.F. Blake, Gymnolomia jelsk ii Hieron., G . hirsuta Klatt

E: CA, Prov. San Ignacio:J. C ampos \& Z Z. G aráa 3977, 4497. Ecuador (Tipo).

\section{BASELLACEAE}

\section{Anredera boliviensis (Hauman) Govaerts}

S: Boussingaultia ramosa var. boliviensis Hauman, Boussingaultia boliviensis (Hauman) J.F. Macbride, †A nredera ramosa (Moquin) Eliasson

E: CA, Prov. San Ignacio:J. Campos \& O. Díaz 2351;J. Campos \& P. D íaz 2654; J. Campos\& P. López 4958. AM, CU, TU. Bolivia.

\section{BORAGIN ACEAE}

\section{Tournefortia fuliginosa Kunth}

S: Tournefortia rugosa Willd.

E: CA, Prov. San Ignacio: R. V ásquez et al. 20225. CU, HU. Ecuador, Colombia, Venezuela. 


\section{BRASSICACEAE}

\section{Capparis polyantha Triana \& Planch.}

S: C apparis flex uosa (L.) L. subsp. polyantha (Trana \& Planch.) H.H. Iltis

E: CA, Prov. San Ignacio: R. V ásquez \& R. Rojas 25173. Bolivia, Colombia.

\section{Cardamine flexuosa With.}

S: Cardamine sylvatica Bess, C. pratensis Hook.f., C. tenuifolia Hook.

E: CA, Prov. San Ignacio:J. Campos \& S. Corrales 3798. Cosmopolita.

\section{BROME LIACEAE}

28. Guzmania pennellii L. B. Sm.

S: Theophyllum pennellii (L. B. Sm.) Mez

E: CA, Prov. San Ignacio: J. Campos et al. 5582. Colombia (Tipo), Ecuador.

\section{Pitcairnia brachyspermaAndré}

E: CA, Prov. San Ignacio: R. V ásquez et al. 26740. Colombia, Ecuador.

30. * Puya robin-fosteriG.S. Varad. \& H. Luther, Selbyana 16(2): 235, f. 2a-d. 1995

E: PA, Prov. Oxapampa: R.B. Foster 9062 (Holótipo: SEL); B. L eón \& K.R. Young 1776 (USM),.Endémica.

31. *Puya tyleriana Sagást., Zapata \& M.O. Dillon, Arnaldoa 11(2): 29-36. 2004

E: AN, Prov. Corongo: A . Sagástegui et al. 17095 (Holótipo: $\mathrm{HAO}$; Isótipos: BM, F, NY, US). Endémica.

32. Tillandsia acuminata L. B. Sm.

E: CA, Prov. San Ignacio: J. Campos et al. 2786. Colombia (Tipo), Ecuador.

\section{Vriesea chontalensis (Baker) L.B. Sm.}

B: Tillandsia chontalensis Baker

S: Tillandsia spuria Mez. \& Werkle

E: CA, Prov. San Ignacio: C. D íaz \& A . Torres 7823. Ecuador, Costa Rica, Nicaragua, Panamá.

\section{CAMPAN ULACEAE}

34. Centropogon quebradanus E. Wimm.

E: CA, Prov. San Ignacio: J. Campos et al. 5475. Ecuador(Tipo).

\section{CUCURBITACEAE}

\section{Cucurbita ficifolia Bouché}

E: CA, Prov. Contumazá: A . Sagástegui \& S. L ópez 10585 (HUT); SM, Prov. Mariscal Cáceres: K. Young \& B. L eón 4988 (HUT).
36. Cucurbita moschata Duchesne

E: AM, Prov. Bagua: V . H odges \& J. G orham 114 (HUT, MO).

Nota: Estas especies nativas no fueron incluidas por Brako \& Zarucchi (1993) y Ulloa Ulloa et al. (2004). La determinación fue efectuada porT. Andres (The Cucurbit Network).

\section{CYPERACEAE}

\section{Fuirena umbellata Rottb.}

S: Scirpus umbellatus (Rottb.) Kuntze

E: CA, Prov. San Ignacio:J. Campos \& 0. D íaz 2075. Cosmopolita.

\section{ERICACEAE}

38. Anthopterus schultzeae(Sleumer) Luteyn

B: Themistodesia schultzeae Sleumer

S: †Themistodesia cutucuensis A.C. Sm.

E: AM, Prov. Bagua: R. V ásquez et al. 26056; R. Rojas et al. 376; CA, Prov. San Ignacio: E . Rodríguez \& J. C ampos 1824. SM. Ecuador (Tipo Basónimo).

Nota: En Brako \& Zarucchi (1993) se indica a T hemistodesia cutuauensis A.C. Sm. como especie no reconfirmada para Perú.

\section{Cavendishia laurifolia (Klotzsch.) Benth. \&} Hook

B: Polyboea laurifolia Klotzsch.

S: Cavendishia tuerckheimii, Chupalon laurifolium (Klotzsch.) Kuntze

E: CA, Prov. San Ignacio: C. D iaz \& A . Torres 7816. Belice, Colombia, Guatemala (Tipo), México, Panamá.

40. Satyria arborea A. C. Sm.

E: CA, Prov. San Ignacio: C. D íaz \& A . Torres 7767. Colombia (Tipo).

\section{FABACEAE}

41. Geoffroea spinosa Jacq.

S: $\mathrm{G}$ eoffroea bredemeyeri Kunth, $\mathrm{G}$ eoffroea superba Bonpl., †G eoffroea striata (Willd.) Morong., Robinia striata Willd.

E: CA, Prov. San Ignacio: E . Rodríguez \& J. C ampos 1691; J. Campos \& E. Rodríguez 4137. PI, TU.

\section{FLACOURTIACEAE}

\section{Casearia quinduensis Tul.}

E: CA, Prov. San Ignacio: J. Campos \& Z. G arcía 3958; J. Campos \& P. D íaz 4368. Ecuador, Colombia (tipo).

43. *Neosprucea rimachi McDaniel ex Alford, Grández \& Vásquez, Arnaldoa 11(2): 37-42, f.1. 2004 [2005].

E: LO, Prov. Maynas: $M$ cD aniel \& Rimachi 20402 (Holótipo: AMAZ, Isótipos: IBE, MO, NY, US, USM, WIS). Colombia. 
44. Xylosma paucinervosa (Steyerm.) Sleumer

B: Banara paucinervosa Steyerm.

E: CA, Prov. San Ignacio: E . Rodríguez 1249; R. V ásquez etal. 20184; R.V ásquez \& J. Campos 26215; J. Campos \& E . Rodríguez 2856; J. Campos \& S. Corrales 3477; J. Campos et al. 6267; C. D íaz \& A. Torres 7759, 7762. Bolivia, Colombia, EcuadorVenezuela (tipo).

\section{GESNERIACEAE}

\section{Besleria angustiflora Fritsch}

E: CA, Prov. San Ignacio: J. Campos \& S. Corrales 3555. Colombia (Tipo), Ecuador.

\section{Besleria modica C. V. Morton}

E: CA, Prov. San Ignacio:J. Campos \& Z. G arća 4455. AM: E . Rodríguez \& J. Rodríguez 806. Ecuador (tipo).

\section{Gasteranthus pansamalanus (Donn. Sm.) Wiehler \\ B: Besleria pansamalana D onn. Sm. \\ E: CA, Prov. San Ignacio: E. Rodríguez \& P. Reyes 1727; J. Campos et al. 4219. Colombia, Ecuador, Guatemala, México.}

48. *Monopyle macrocarpa Benth, Icon. PI. 12: 8586, t. 1198, f.1-5. 1876.

E: SM, Prov. San Martín (Tarapoto): Spruce 4151 (Holótipo: K, Isótipos: BR, W), CA, Prov. San Ignacio: V . Q uipuscoa 393. AM, HU, MD..Colombia, Ecuador, Costa Rica, Panamá.

Nota: Esta especie fue descrita con material peruano en 1876 y no fue incluida por Brako \& Z arucchi (1993) ni Ulloa Ulloa et al. (2004).

\section{GROSSULARIACEAE}

49. *Ribes amazonica Weigend \& E. Rodr., Arnaldoa 12(1-2): 42-47, f. 1a-h, 2a-c. 2005 [2006].

E: AM, Prov. Chachapoyas: M. Weigend et al. 98/355 (Holótipo: USM, Isótipos: CPUN, F, M).

50. ${ }^{\star}$ Ribes colandina Weigend, Rev. peru. biol. 12(2): 263-265, f. 7a-c, 8a-g. 2005.

E: LL, Prov. Bolívar: M. W eigend et al. 2000/ 823 (Holótipo: USM, Isótipos: HUT, M, B); CA: Prov. San Miguel de Pallaques: E. Rodríguez et al. 2288 (F, HUT, M, MO, NY), 2310 (F, HUT, MO, NY), E . Rodríguez et al. 2359 (F, HUT, M, NY), E . Rodríguez et al. 2367 (HUT, MO, NY); PI, Prov. Ayabaca: C. D íaz \& R. V ásquez 3003 (MO). AM, LA.

\section{LAMIACEAE}

51. $\ddagger$ Clinopodium nubigenum (Kunth) Kuntze

B: Thymus nubigenus Kunth

S: Micromeria nubigena (Kunth) Benth., †Satureja nubigena (Kunth) Briq.
E: AM, Prov. Chachapoyas: A . Sagástegui 7479 (HUT); CA, Cajamarca: A . Sagástegui 8122 (HUT, MO), Prov. Contumazá: A. Sagástegui 14002 (HUT), Prov. San Miguel de Pallaques: A . Sagástegui et al. 9535 (HUT, MO), Prov. Celendín: A . Sagástegui et al. 12277 (HUT). LA, Prov. Ferreñafe: A . Sagástegui et al. 12844 (F, HUT, MO ). LL, Prov. Bolivar: A . L ópez \& A . Sagástegui 3268 (HUT); E . Rodríguez \& V . M edina 2600 (HUT). SM, Prov. Mariscal Cáceres: K. Young \& B. L eón 4837 (F, HUT). Colombia, Ecuador.

\section{LAURACEAE}

\section{2. *Endlicheria arachnocome Chanderbali, $\mathrm{Fl}$.} Neotrop. 91:52. 2004

E: LO, Prov. Maynas: M. Rimachi 10532 (Holótipo: MO, Isótipo: NY), R.V ásquez \& N. Jaramillo 3157, 5253, 5457, 7620, 7647, 8414; R. V ásquez et al. 5434; Prov. Requena: R.V ásquez \& Jaramillo 8766. Endémica.

53. ${ }^{\star}$ Endlicheria argentea Chanderbali, Fl. Neotrop. 91: 110-112, f.40, 41.2004

E: LO, Prov. Ucayali (Sapuena, Jenaro Herrera): R.V ásquez \& R. Jong 12397 (Holótipo: MO, Isótipo: MO). Colombia.

\section{Endlicheria canescens Chanderbali}

E: CA, Prov. San Ignacio: E. Rodríguez \& R. Cruz 2039; J. Campos\& O. Cano 4695. LO. Brasil, Ecuador, Colombia, Surinam (tipo), Venezuela.

\section{5. *Endlicheria duotincta Chanderbali, $\mathrm{Fl}$. Neotropic. 91: 90-92, f.30, 31. 2004}

E: CA, Prov. San Ignacio: E. Rodríguez \& P. Reyes 1807 (Holótipo: MO ; Isótipos: F, G, HBG, HUT, NY, US, U). SM. Ecuador.

\section{Endlicheria griseo-sericea Chanderbali}

E: CA, Prov. San Ignacio: R.V ásquez \& S. Flores 26313. AM, PA, SM. Ecuador (Tipo), Colombia.

57. ${ }^{\star}$ Endlicheria oreocola Chanderbali, FI. Neotrop. 91: 86-88, f.27, 29. 2004

E: CA, Prov. San Ignacio: J. Campos \& S. N uñez 4669 (Holótipo: MO, Isótipos: F, HBG, HUT, NY); J. Campos et al. 5902, 5915. Ecuador.

58. *Endlicheria tomentosa Chanderbali, $\mathrm{Fl}$. Neotropic. 91: 48-51, f. 14,15. 2004

E: CA, Prov. San Ignacio: J. Campos et al. 2938 (Holótipo: MO, Isótipos: F, G, HBG, HUT, NY, MO, U). Endémica.

59. Mutisiopersea mutisii (Kunth) Kosterm.

B: Persea mutisii Kunth

E: CA, Prov. San Ignacio: J. C ampos et al. 5653, 5838. Colombia, Ecuador.

Nota: En Brako \& Zarucchi (1993) se indicaa [P ersea mutisii Kunth] como no conocida de Perú.

60. Ocotea infrafoveolatavan der Werff 
E: CA, Prov. San Ignacio:J. Campos et al. 5577; M . H uamán et al. 022, 023, 055. Ecuador (tipo), Colombia.

\section{Rhodostemonodaphne praeclara(Sandwith) Madriñan}

B: N ectandra praeclara Sandwith

E: AM, Prov. Bagua: R. V ásquez et al 23876; CA, Prov. San Ignacio: J. Campos \& R. V ásquez 6410. Brasil, Ecuador, Guyana Británica, Venezuela.

\section{LOASACEAE}

62. *Nasa longivalvis E. Rodr. \& Weigend, Arnaldoa 11(2): 67-77. f 2a-f, 3a-j. 2004 [2005].

E: LL, Prov. Bolivar: E . Rodríguez \& V . M edina 2557 (Holótipo: HUT; Isótipos: BSB, F, HAO, MO, USM); E . Rodríguez \& V . M edina 2559, 2610; Prov. Sánchez Carrión: M. W eigend \& Ch. Schwarzer 7910.

\section{MALVACEAE}

63. Luehea candicans Mart.

E: CA, Prov. San Ignacio: C. D íaz 2042. Bolivia, Brasil (tipo), Paraguay, Venezuela.

\section{Byttneria minytricha Cristóbal}

E: CA, Prov. San Ignacio: J. Campos \& Z. Garća 4006, 4501. Ecuador (tipo).

\section{Hibiscus diversifolius Jacq.}

E: CA, Prov. San Ignacio: E . Rodríguez \& J. Campos 1846; J. Campos \& O. D íaz 2363; J. Campos \& M. L ópez 2590. Brasil, Costa Rica, Ecuador, Honduras, Africa y Madagascar.

66. *Hibiscus chancoae Krapov. \& Fryxell, Bonplandia (Corrientes) 13(1-4): 58, f. 6-9. 2004.

E: SM, Prov. Huallaga, La Morada, margen del Rio Huallaga: M. Chano E . 564 (Holótipo: CTES, Isótipo: USM, n.v.)

\section{7. $\quad \ddagger$ Pseudabutilon umbellatum (L.) Fryxell}

S: $†$ A butilon umbellatum (L.) Sweet, Sida umbellata L., A butilon andersonianum $\mathrm{G}$ arcke

E: CA, Prov. San Ignacio: E . Rodríguez 1221; J. Campos \& 0. D íaz 2500. América Central (Costa Rica, México, Honduras).

\section{MONIMIACEAE}

68. Siparuna harlingii S.S. Renner \& Hausner

E: CA, Prov. San Ignacio: E. Rodríguez \& P. Reyes 1745; J. Campos \& S. N uñez 4630; AM, Prov. Bagua: $H$. van der $W$ erff, $R$. V ásquez \& B. G ray 16280. Ecuador (Tipo), Colombia.

\section{MORACEAE}

69. Ficus coerulescens (Rusby) Rossberg

S: U rostigma coerulescens Rusby

E: CA, Prov. San Ignacio: R. V ásquez \& R. Rojas 25169; J.
Campos et al. 3200. LO, MD. Bolivia, Colombia, Ecuador.

\section{Ficus trapezicola Dugand}

E: CA, Prov. San Ignacio: R. V ásquez et al 26567;J. Campos \& M. L ópez 2581; J. Campos \& E . Rodríguez 2829; J. Campos et al. 6256. AM. Colombia.

\section{ONAGRACEAE}

\section{Fuchsia putumayensis Munz}

E: CA, Prov. San Ignacio: E . Rodríguez 1974; J. Campos \& P. L ópez 4945. Colombia, Ecuador.

\section{O RCH IDACEAE}

Nota: Las colecciones de G. Calatayud han sido depositadas sólo en CUZ y USM.

\section{Brachionidium ephemerum Luer}

E: CA, Prov. San Ignacio: G . Calatayud et al. 714. Ecuador (Tipo).

\section{Catasetum imperiale L. Linden \& Cogn.}

E: CA, Prov. San Ignacio: G . C alatayud etal. 836; G . C alatayud 916.

\section{Chrysocycnis lehmannii Rolfe}

E: CA, Prov. San Ignacio: R. V ásquez et al. 26175. Colombia, Ecuador.

\section{5. $\neq$ Crocodeilanthe salpingantha (Luer \& Hirtz) Luer}

S: †Pleurothallis salpingantha Luer \& Hirtz, Stelis salpingantha (Luer \& Hirtz) Pridgeon \& M.W. Chase

E: CA, Prov. San Ignacio: G . Calatayud et al. 440. Ecuador (Tipo).

\section{Cranichis polyantha Schltr.}

S: C ranichis calva var. vargasii C. Schweinf., C. polyantha var. caqetaensis Renz

E: CA, Prov. San Ignacio: G . C alatayud et al. 398, 443. Ecuador. Perú.

Nota: En Brako \& Zarucchi (1993) como no conocida de

\section{Dichaea dammeriana Kraenzl.}

E: CA, Prov. San Ignacio: G . Calatayud et al. 307, 333. Costa Rica, Nicaragua, Panamá, Ecuador.

\section{Dichaea histrio Rchb. F.}

E: CA, Prov. San Ignacio: G . Calatayud et al. 391. Colombia, Ecuador.

\section{Dichaea swartzii (C. Schweinf.) Garay \& H. R.} Sweet

B: D idhaea pendula (Aublet) Cogniaux var. swartzii C. Schweinf.

S: Cymbidium echininocarpum (Sw.) Willd.; E pidendrum 
echinocarpon Sw.

E: CA, Prov. San Ignacio: G . Calatayud et al. 317. Colombia, Colombia, Venezuela.

80. Dryadella minuscula Luer \& R. Escobar

E: CA, Prov. San Ignacio: G . Calatayud et al. 552. Colombia.

81. Elleanthus ampliflorus Schltr.

E: CA, Prov. San Ignacio: G . Calatayud et al. 274, 461. Ecuador.

\section{Elleanthus blatteus Garay}

E: CA, Prov. San Ignacio: G . Calatayud et al. 578, 628. Ecuador (Tipo).

\section{Epidendrum secundum Jacq.}

S: E pidendrum elongatum Jacq.; A mphiglottis seaunda (Jacq.) Salisb.

E: CA, Prov. San Ignacio: G . C alatayud et al. 452, 568, 637, 670, 671, 672, 739, 813, 824, 834, 842; E . Rodríguez 1844. AM, HU, JU, LO, PA, PI, SM, TU. Bolivia, Brasil, Colombia, Ecuador, Venezuela.

84. *Epidendrum stenocalymmum Hágsater \& Calatayud, Icon. Orchid. (Mexico) 7(4): t. 790. 2004

E: CA, Prov. San Ignacio: J. Campos et al. 5538 (Holótipo: USM; Isótipo: AMO ); G. C alatayud et al. 279; G . Calatayud et al. 658; D íaz et al. 10470. Perú. Endémica.

85. Exalaria parviflora (Presl) Garay \& G.A. Romero

B: 0 phrys parviflora Presl

S: †Cranichis fertilis (Lehman \& K raezl.) Schlechter

E: CA, Prov. San Ignacio: G . Calatayud et al 556, 575. HU, SM.

86. Gomphichis adnata (Ridl.) Schltr.

B: Stenoptera adnata Ridl.

E: CA, Prov. San Ignacio: G . Calatayud et al. 271, 717. Colombia.

\section{Gongora gracilis Jenny}

E: CA, Prov. San Ignacio: G. Calatayud et al. 688. Ecuador (Tipo).

\section{Lepanthes erepsis Luer \& Hirtz}

E: CA, Prov. San Ignacio: G. Calatayud et al. 258, 768. Ecuador (Tipo).

\section{Masdevallia odontopetala Luer}

E: CA, Prov. San Ignacio: G . Calatayud et al. 595, 631, 675, 729. Ecuador (Tipo).

90. Maxillaria brevifolia (Lindl.) Rchb. f.

B: Camaridium brevifolium Lindl.

E: CA, Prov. San Ignacio: G. Calatayud et al. 418, 763. PA.
Bolivia, Ecuador, Colombia.

\section{Maxillaria guareimensis Rchb.f.}

E: CA, Prov. San Ignacio: G. Calatayud et al. 235. Bolivia, Ecuador, Venezuela (Tipo).

92. Maxillaria ochracea (Rchb. f.) Garay

S: Ornithidium ochraceum Rchb.f.

E: CA, Prov. San Ignacio: G . Calatayud, et al. 906.

93. Maxillaria pachyneura F. Lehm. \& Kraenzl.

E: CA, Prov. San Ignacio: G . Calatayud et al. 666, 682, 734, 765, 786. Ecuador (Tipo).

94. Maxillaria stenophylla Rchb. f.

E: CA, Prov. San Ignacio: G. Calatayud et al. 410. Bolivia, Colombia, Ecuador, Venezuela (Tipo).

\section{Octomeria callosa Luer}

E: CA, Prov. San Ignacio: G . Calatayud et al. 280, 652. Ecuador (Tipo).

96. Oncidium abortivum Rchb. $\mathrm{f}$.

E: CA, Prov. San Ignacio: G . C alatayud et al. 584, 645. Colombia (Tipo), Ecuador.

\section{Pleurothallis alveolata Luer}

E: CA, Prov. San Ignacio: G. Calatayud et al 693. Ecuador (Tipo).

\section{Pleurothallis brachiata Luer}

E: CA, Prov. San Ignacio: G . Calatayud et al. 564, 599, 619. Ecuador (Tipo).

\section{Pleurothallis cardiantha Rchb. $f$.}

E: CA, Prov. San Ignacio: G . C alatayud et al. 344, 563, 686, 724, 778. Ecuador, Venezuela.

100. Pleurothallis cardiostola Rchb.f.

E: CA, Prov. San Ignacio: G. Calatayud et al 373. Ecuador.

Nota: En Brako \& Zanucchi (1993) estácomo no reconfirmado para Perú.

101. Pleurothallis cernua Luer

E: CA, Prov. San Ignacio: G . Calatayud et al 253. Ecuador (Tipo).

102. Pleurothallis cordifolia Richb.f. \& Wagener

E: CA, Prov. San Ignacio: G . C alatayud et al 345, 370, 372, 422, 444. Colombia, Ecuador, Venezuela (Tipo).

103. Pleurothallis lemniscifolia Luer

E: CA, Prov. San Ignacio: G . C alatayud et al. 350, 352, 402. Ecuador(Tipo).

104. Pleurothallis Iilijae Foldats 
E: CA, Prov. San Ignacio: G . C alatayud et al. 249. Ecuador, Venezuela(Tipo).

\section{Pleurothallis stenosepala Rolfe}

E: CA, Prov. San Ignacio: G . C alatayud et al. 346, 364, 394, 598. Ecuador.

\section{Pleurothallis phyllocardioides Schltr.}

E: CA, Prov. San Ignacio: G . C alatayud et al. 251, 566, 597. CU, HU. Bolivia, Colombia, Costa Rica, Ecuador (Tipo), Panamá.

107. $\ddagger$ Prosthechea aemula (Lindl.) W.E. Higgins

B: E pidendrum aemulum Lindl.

S: †E ncydia aemula (Lindl.) Carnevali \& I. Ramírez, E ncyclia fragans subsp. aemula (Lindl.) D ressler, E pidendrum fragans var. aemulum (Lindl.) Barbosa Rodr., E pidendrum lineatum Salisb.

E: CA, Prov. San Ignacio: G . Calatayud et al. 668; R. V ásquez et al. 25120; J. Campos \& P. Díaz 2700. AM. HU, LO. Ecuador, Panamá

108. $\ddagger$ Prosthechea hartwegii (Lindl.) W.E. Higgins

B: $\mathrm{E}$ pidendrum hartwegii Lindl.

S: †E ncydia hartwegii (Lindl.) Vásquez \& D odson

E: CA, Prov. San Ignacio: G . Calatayud et al 573. CU, JU. Ecuador.

109. $\ddagger$ Prosthechea pygmaea (Hook.) W.E. Higgins

B: E pidendrum pygmaeum Hook.

S: †E ncydia pygmaea (Hook.) D ressler

E: CA, Prov. San Ignacio: G . C alatayud et al 560, 593. Colombia, Ecuador, Mesoamérica.

110. Scaphyglottis bicornis (Lindl.) Garay

B: $\mathrm{H}$ ex adesmia biornis Lindl.

S: H ex isea biomis (Lindl.) D ressler

E: CA, Prov. San Ignacio: G . Calatayud et al. 250, 445. Colombia, Ecuador, Venezuela.

111. Specklinia fornicata (Luer) Luer

B: Pleurothallis fornicata Luer

S: Stelis fornicata (Luer) Pridgeon \& M.W. Chase

E: CA, Prov. San Ignacio: G . C alatayud et al. 585, 601, 730, 896. AM. Ecuador (Tipo del Basónimo).

112. Stelis pichinchae Dodson \& Garay

E: CA, Prov. San Ignacio: G . Calatayud et al 273, 332. Ecuador (Tipo).

113. Stelis spathulata Poepp. \& Endl.

E: CA, Prov. San Ignacio: G . Calatayud 777. Colombia, Costa Rica, Ecuador, Panamá, Venezuela.
114. Trichosalpinx apoda (Garay \& Dunst.) Luer

B: Pleurothallis apoda G aray \& D unst.

E: CA, Prov. San Ignacio: G . C alatayud et al. 769. Ecuador, Venezuela (Tipo del Basónimo).

\section{Trichosalpinx chaetoglossa (Luer) Luer}

B: Pleurothallis chaetoglossa Luer

E: CA, Prov. San Ignacio: G . Calatayud et al. 747. Ecuador (Tipo del Basónimo).

\section{PLUMBAGINACEAE}

\section{Plumbago zeylanica $L$.}

E: CA, Prov. San Ignacio:J. Campos \& O. D íaz 2155. Cosmopolita.

\section{POACEAE}

117. Gouinia paraguayensis (Kuntze) Parodi var. paraguayensis

B: A rundinaria paraguayensis K untze

E: CA, Prov. San Ignacio: J. Campos \& O. D íaz 2269. Bolivia, Paraguay (Tipo del Basónimo).

118. Lasiacis ruscifolia (Kunth) Hitch.

B: Paniaum ruscifolium Kunth

E: CA, Prov. San Ignacio: S. L latas et al 9175. México (Tipo del Basónimo).

119. Setaria sphacelata (Schumach.) M.B. Moss ex Stapf \& C.E. Hubb

B: Paniaum sphacelatum Schumach.

E: CA, Prov. San Ignacio: J. Campos \& W. V argas 3905; S. Flores \& E . D elgado 219. Bolivia, Ecuador, Costa Rica, México. África \& Madagascar.

\section{PROTEACEAE}

120. Panopsis perijensis Steyerm. ex K. S. Edwards E: CA, Prov. San Ignacio: J. C ampos et al. 5886, 5962; C. D íaz \& S. Fernández 10197. Colombia, Ecuador, Venezuela(Tipo).

\section{RUBIACEAE}

\section{Ladenbergia chapadensis $S$. Moore}

E: CA, Prov. San Ignacio:J. Campos\& Z . G aráa 3956A . Brasil (Tipo).

122. Psychotria cuspidata Bredem. ex Roem. \& Schult.

E: CA, Prov. San Ignacio: R. V ásquez et al. 26684;J. Campos \& S. N úñez 4597. AM, LO, PA. Ecuador, Colombia, Venezuela (Tipo).

Nota: Especies determinadas por C. M. Taylor (MO).

\section{RUTACEAE}




\section{Zanthoxylum quinduense Tul.}

S: Fagara quinduense(Tul.) Engl., Fagara macosperma (Tul.) Engl., Zanthox ylum macrospermum Tul.

E: CA, Prov. San Ignacio: R. V ásquez et al. 20172; C . D íaz \& A . Torres 7824. AM, LO, PI. Colombia(Tipo), Ecuador, Venezuela.

\section{SAPIN DACEAE}

\section{Cupania americana $L$.}

E: CA, Prov. San Ignacio: R. V ásquez \& A . V ásquez 20893. Ecuador, Bolivia, Colombia, Venezuela.

Nota: R.V ásquez \& A .V ásquez 20883 de la misma localidad anterior, ha sido determinada como Cupania americana L. subsp. glandulosa T.D. Penn. por R. Vásquez (MO). Este taxón ha sido expuesto como nom. nud. por Vásquez et al. (2002) para el D pto. Amazonas.

\section{SOLANACEAE}

\section{Nicotiana tomentosiformis Goodsp.}

E: CU, Prov. La Convención: G . C alatayud et al. 1446 (CUZ, MO). Bolivia (Tipo).

\section{Sessea macrophylla Francey}

E: CA, Prov. San Ignacio: E. Rodríguez 645; E. Rodríguez \& S. L eiva 2104; J. C ampos \& 0. D iaz 2303. HU, JU. Ecuador

\section{Sessea colombiana Francey}

E: CA, Prov. Santa Cruz: Santisteban \& G uevara 136 (F, HUT). Bolivia, Colombia (Tipo), Venezuela.

\section{Solanum pachyandrum Bitter}

E: CA, Prov. San Ignacio: E. Rodríguez et al. 1607;j. Campos et al. 2806. Ecuador (Tipo).

\section{URTICACEAE}

\section{Urtica longispica Killip}

E: CA, Prov. San Miguel de Pallaques: E. Rodríguez et al. 2726 (HUT); M . W eigend et al. 7485 (B, HUT, USM, M); M . W eigend et al. 7488 (B, HUT, USM). Colombia, Ecuador.

Nota: Especie determinada por M. Weigend (MSB).

\section{VISCACEAE}

\section{Dendrophthora luerii Kuijt}

E: AM, Prov. Bongará: R. V ásquez etal. 27668; CA, Prov. San Ignacio: J. C ampos et al. 5569. Colombia, Ecuador (Tipo).

\section{ZIN GIBE RACEAE}

\section{Renealmia fragilis Maas}

E: CA, Prov. San Ignacio: E. Rodríguez 689. LO. Colombia, Ecuador (Tipo).

\section{Agradecimientos}

Los autores agradecen a laJohn D. and Catherine T. M acA rthur Foundation por brindar el apoyo económico para este estudio, al Missouri Botanical G arden (MO) y al Herbarium Truxillense (HUT) instituciones cooperantes, así como al INRENA porlos diversos permisos de colección. A los directores y curadores de los siguientes herbarios por brindarnos información o permitirnos el acceso a sus colecciones: F, HAO, HUT, NY, MO, QCNE, US y USM. A los D rs. A. Sagástegui (HAO), M. O. Dillon (F), D. Neill (MO, QCNE), C. Ulloa Ulloa (MO), S. Knapp (BM), N. Hensold (ex F), M. Weigend (BSB), A. Cano (USM), M. Chanco (USM), S. Leiva (HAO), M. Zapata (HAO), V. Q uipuscoa (HUSA) y S. Llatas (PRG) por proporcionarnos información de las especies del área o por sus magníficas sugerencias. Un reconocimiento especial a los diferentes especialistas que han intervenido en la determinación específica: $M$. Weigend (BSB), T. Andres (The Cucurbit Network), M. Alford (BH), H. Balslev, A. Chanderbali (MO ), G. D avidse (MO), E. Hágsater (AMO), M. Nee (NY), E. Christenson, D. Bennet, J. Kuijt (LEA), M. Pirie (U), P. Fryxell (TEX), H. Luther (SEL), L.E. Skog (US), C. Luer (MO), C. Benítez (MY), C. Cristóbal (CETES) y C.C. Berg (BG). También, agradecemos sinceramente a los colectores y asistentes de campo 1993-2002 y a todos aquellos anónimos que colaboraron en la ejecución general del Proyecto.

\section{Literatura Citada}

APG. 2003. An update of the Angisoperm Phylogeny Group classification for the orders and familias of flowering plants: APG II. Botanical Journal of the Linnean Society, 141: 399-436

Brako, L. \& J. Zarucchi. 1993. Catálogo de las Angiospermas y Gimnospermas del Perú. Monogr. Syst. Bot. Missouri Bot. Garden. Vol 45.

Holmgren P., N. H. Holmgren \& L. C. Barnett. 1990. Index Herbariorum. Part I, The Herbaria of the world, $8^{\text {th }}$ edition. New York Botanical Garden. New York, NY, U.S.A.

Judd W. S., et al. 1999. Plant Systematics: A Phylogenetic Approach. Sinauer Associates, INC. Sunderland, Massachusetts, USA.

Judd W. S., et al. 2002. Plant Systematics: A Phylogenetic Approach. $2^{\text {nd }}$ edn. Sinauer Associates, INC. Sunderland, Massachusetts, USA.

Rodríguez E., R. Vásquez, R. Rojas \& J. Campos. 2004. Nuevas Adiciones de Angiospermas a la Flora Peruana procedentes de la Provincia de San Ignacio, Dpto. Cajamarca. En Libro de Resúmenes del X Congreso Nacional de Botánica, 2 - 5 Mayo 2004, Trujillo - Perú. Pág. 157.

Ulloa Ulloa C., J. L. Zarucchi \& B. León. 2004. Diez años de Adiciones a la Flora del Perú: 1993-2003. Arnaldoa (Edic. Esp. Noviembre 2004): 1-242

Vásquez R., 1997. Flórula de las Reservas Biológicas de Iquitos, Perú. Allpahuayo Mishana Explornapo Camp Explorama Lodge. Monogr. Syst. Bot. Missouri Bot. Garden. Vol 63.

Vásquez R., R. Rojas, C. Díaz \& E. Rodríguez. 1998. Catálogo Preliminar de las Plantas Vasculares de la Cuenca del Río Cenepa y Adyacentes. Amazonas - Perú. En Libro de Resúmenes del VII Congreso Nacional de Botánica, 25 30 Mayo 1998, Cajamarca - Perú. Pág. 178. 
Vásquez R., R. Rojas, C. Díaz \& E. Rodríguez. 1999. Diversidad Florística del Río Cenepa y Áreas Adyacentes, Amazonas - Perú. International Botanic Congress IBC99, St. Louis-USA. Disponible en CD-ROM.

Vásquez R., R. Rojas \& E. Rodríguez. 2002a. Adiciones a la Flora peruana: especies nuevas, nuevos registros y estados taxonómicos de las Angiospermas para el Perú. En Libro de Resúmenes del IX Congreso Nacional de Botánica, 17 - 22 Mayo 2002, Iquitos - Perú. Pág. 69.

Vásquez R., R. Rojas \& E. Rodríguez. 2002b. Adiciones a la Flora peruana: especies nuevas, nuevos registros y estados taxonómicos de las Angiospermas para el Perú. Arnaldoa 9(2): 43-110.

Weigend M. 2002. Observations on the Biogeography of the Amotape-Huancabamba Zone in Northern Peru. In: K. Young et al., Plant Evolution and Endemism in Andean South America. Bot. Review 68(1): 38-54.

Weigend M. 2004. Additional observations on the biogeography of the Amotape-Huancabamba zone in Northern Peru: Defining the South-Eastern limits. Rev. Peru. Biol. 11(2): 127-134. 